\title{
Polarization-balanced design of heterostructures: Application to AIN/GaN double-barrier structures
}

\author{
Kristian Berland, Thorvald G. Andersson, and Per Hyldgaard \\ Department of Microtechnology and Nanoscience, MC2, Chalmers University of Technology, SE-41296 Göteborg, Sweden
}

(Received 23 October 2011; published 16 December 2011)

\begin{abstract}
Inversion and depletion regions generally form at the interfaces between doped leads (cladding layers) and the active region of polar heterostructures like $\mathrm{AlN} / \mathrm{GaN}$ and other nitride compounds. The band bending in the depletion region sets up a barrier that may seriously impede perpendicular electronic transport. This may ruin the performance of devices such as quantum-cascade lasers and resonant-tunneling diodes. Here we introduce the concepts of polarization balance and polarization-balanced designs: A structure is polarization balanced when the applied bias match the voltage drop arising from spontaneous and piezeolectric fields. Devices designed to operate at this bias have polarization-balanced designs. These concepts offer a systematic approach to avoid the formation of depletion regions. As a test case, we consider the design of AlN/GaN double-barrier structures with $\mathrm{Al}_{\tilde{x}} \mathrm{Ga}_{1-\tilde{x}} \mathrm{~N}$ leads. To guide our efforts, we derive a simple relation between the intrinsic voltage drop arising from polar effects, average alloy composition of the active region, and the alloy concentration of the leads. Polarization-balanced designs secure good filling of the ground state for unbiased structures, while for biased structures with efficient emptying of the active region they remove the depletion barriers.
\end{abstract}

DOI: 10.1103/PhysRevB.84.245313

PACS number(s): 73.21.-b, 73.63.-b, 78.67.-n, 61.46.-w

\section{INTRODUCTION}

Heterostructures of aluminium nitride (AIN) and gallium nitride $(\mathrm{GaN})$ are key material systems for novel optoelectronic devices. ${ }^{1,2}$ The large band offset $(\sim 2 \mathrm{eV})$ and direct-band gap permit intersubband transitions in the near infrared, ${ }^{3-7}$ which makes them attractive for building devices working at fiberoptic wavelengths. They hold the promise of extending the operational regime of quantum cascade (QC) lasers in both the near and the far infrared. ${ }^{8-11}$ The latter are due to the large longitudinal-optical (LO)-phonon energy $\omega_{\mathrm{LO}} \sim 90 \mathrm{meV}$. For wurtzite AlN/GaN structures grown in the $c$-plane direction, the band profile is distinguished by huge internal electrical fields. These fields arise from the interface charges originating in spontaneous and piezoelectric polarization. ${ }^{12,13}$

AlN/GaN intersubband devices offer great opportunities, but challenges remain both for growth and design. Interface roughness, ${ }^{14}$ high dislocation density, and scarcity of highquality substrates limit device performance. Moreover, precise control of multiple quantum levels and efficient transport through the structure demand ultrathin AlN layers. This demand arises because the large band offset and heavy conduction electron mass $\sim(0.2-0.3) m_{0}$ weaken the coupling between states in adjacent quantum wells. The wide depletion regions and two-dimensional electron gases (2DEGs) forming at the two sides of an active region ${ }^{2,4,15-17}$ exacerbates this challenge for devices based on perpendicular quantum transport. For instance, in resonant-tunneling diodes and QC lasers, the depletion regions make it difficult to simultaneously obtain efficient current injection and control over the alignment of levels and transitions in the structure. Presently, current-driven devices are mostly limited to resonant-tunneling diodes, often exhibiting large hysteresis. ${ }^{14,18-23}$ A related issue exists for structures operating at zero bias (like absorption structures), especially those of only a few layers: As electrons accumulate in the $2 \mathrm{DEG}$, the active region is depleted. ${ }^{2,4,16,17}$

To tackle some of the design problems, we here introduce the two related concepts of polarization balance and polarization-balanced design. A structure is polarization balanced when the unscreened field vanishes in the cladding layers (buffer and cap) layers. We will denote these regions as leads if the structure is intended for perpendicular transport. By unscreened we mean the field that arises from the polarizationinduced interface charges $\sigma_{\text {pol }}$ and from the applied bias $V_{\text {bias }}$, including dielectric screening by valence electrons but excluding screening by conduction electrons and donors. The concept is similar in spirit to strain balance. ${ }^{24}$ The electrical fields in the leads (cladding layers) generally bring about depletion and inversion regions outside the active region. Since the band tailing in the depletion region impedes electron transport, polarization balance helps enable efficient injection into the central active structure. Nonpolar structures are always polarization balanced at zero bias. Many polar structures are polarization balanced at a certain bias.

Polarization-balanced designs are structures tailored to operate at a bias $V_{\text {bias }}^{0}$ resulting in polarization balance. In this study, we rely on alloyed leads to retain design freedom for polarization-balanced designs. A few observations substantiated in this paper clarify the concept and highlight opportunities of such designs. First, these designs can, at a desired operational bias $V_{\text {bias }}^{0} \neq 0$, provide flat bands in the injection region and in the leads in general. There is no depletion barrier. Second, polarization balance at $V_{\text {bias }}^{0}=0$ is also possible by using a suitable choice of composition in the cladding layers. In this case, charge accumulates within the active region. Third, if charge accumulates in the active region-which can also happen for a finite bias, for instance (in a quasistatic picture) when the lowest level of the active region falls close to or below the Fermi level-polarization balance does not result in flat bands. Fourth, a polar device can be operated for a bias before, at, or beyond polarization balance, that is, $\varphi_{\text {bias }}<\varphi_{b}^{0}, \varphi_{b} \approx \varphi_{b}^{0}$, or $\varphi_{b}>\varphi_{b}^{0}$ with $\varphi_{b}=$ $-V_{\text {bias }}$ and $\varphi_{b}^{0}=-V_{\text {bias }}^{0} \cdot{ }^{25}$ Fifth, nonpolar structures cannot operate before and typically must operate much beyond the bias resulting in polarization balance. Sixth, since, in polar structures, current-driven devices such as quantum-cascade lasers can operate at the bias giving polarization balance with 
ensuing flat bands in the injection region, good injection can be ensured without the need of tailoring graded digital alloys in the transport region. ${ }^{26}$ Our calculations of conduction-band profile and quantum states presented here are based on the Schrödinger-Poisson (SP) equation ${ }^{16,27}$ in the effective-mass, envelope-function approximation, including nonparabolicity and exchange-correlation effects as described in Ref. 16.

This paper is presented as follows. The Sec. II discusses polarization-balanced designs. We illustrate the formation of depletion and inversion regions for an AlN/GaN heterostructure; we demonstrate that the depletion barrier seriously inhibits the perpendicular current; and, finally, we take a trial-and-error approach to polarization balance for AlN/GaN double-barrier structures with AlGaN leads of varying alloy concentrations. In Sec. III, we develop a theory to guide polarization-balanced designs. For an active region consisting of $\mathrm{AlN}$ and $\mathrm{GaN}$ layers strained to $\mathrm{Al}_{\tilde{x}} \mathrm{Ga}_{1-\tilde{x}} \mathrm{~N}$ leads, a simple relation between intrinsic voltage drop, alloy concentration $\tilde{x}$, and average $\mathrm{Al}$ composition $x$ in the active region is developed. Section IV contains some simple applications: We, first, demonstrate that choosing a polarization-balance design for an unbiased structure secures good electron filling in the active regions; we then turn to a large parameter-space study of a two-level quantum cascade structure. We find that the use of AlGaN instead of GaN leads significantly increases our ability to realize polarization-balanced designs. The final sections hold our discussions, prospects, and conclusions.

\section{DEPLETION BARRIERS IN ALN/GAN HETEROSTRUCTURES}

That depletion regions form outside the active regions in polar heterostructures is well known. ${ }^{2,15,16,28,29}$ We here observe that these have a huge impact on the function and transport properties of AlN/GaN heterostructures. By calculating the conduction-band profile of a double barrier we, first, illustrate the formation of such a depletion barrier. Then, by calculating the perpendicular current through a single AlN barrier having GaN leads, which allows for a simple and transparent analysis, we make evident how severely this barrier inhibits current injection. Finally, we bring to view how using AlGaN leads rather than $\mathrm{GaN}$ shortens or even removes these depletion regions. Such an approach opens the possibility of avoiding the depletion regions without needing huge biases that may prohibit realistic device design. This result influences the development of our theory and SP calculations in later sections.

\section{A. Depletion and inversion regions}

Interface charges arise at the boundary between different layers of polar heterostructures as follows:

$$
\sigma_{\mathrm{pol}}^{i}=\hat{z} \cdot\left(-\mathbf{P}^{i-1}+\mathbf{P}^{i}\right)=-P^{i-1}+P^{i} .
$$

The conduction band profile produced by these charges exhibits a characteristic sawtoothlike shape for a layered heterostructure..$^{2,13,16,27,30-32}$ If the lead composition and lattice constant is the same on both sides of the active region, so $P_{0}=P_{N}$, the polar contributions to the electrical field vanish in the leads because the net charge produced by the polarization-induced charge in the active structure is zero: $\sum_{i}^{N} \sigma_{\text {pol }}^{i}=0$.

The intrinsic voltage drop over the active structure, arising from the polarization-induced charges $V_{\text {pol }}$, does not, in general, vanish. For a structure in equilibrium $V_{\text {bias }}=0$ some mechanism must compensate for this potential drop, so the Fermi level can remain constant throughout the structure. For a doped structure, the electrical fields rearrange conduction electrons into an inversion (accumulation) region on one side of the structure, leaving behind a depletion (spacecharge) region on the other. This charge transfer results in a potential drop that cancels the intrinsic voltage drop for a structure that is unbiased in equilibrium. If the screening by conduction electrons and ionized donors is so weak that the Fermi-level falls close to the valence band (as for low donor concentration and large potential variations), valence electrons may tunnel into the conduction band, creating holes. Hence, such polarization-induced doping can also compensate for the intrinsic potential drop. ${ }^{33}$ In our study, the formation of depletion and inversion regions in the conduction band is the sole mechanism responsible for securing equilibrium.

Figure 1 illustrates the formation of depletion and inversion regions for an unbiased double-barrier structure, with AIN barriers, and GaN well and cladding layers. The conductionband profile (upper panel) and electron density (lower panel) are obtained in a Schrödinger-Poisson calculation. ${ }^{16}$ We use hard wall boundary conditions far outside the active region (boundaries not displayed) so a proper bulk develops within the cladding layers. The lower panel shows that as electrons accumulate on the left, a wide depletion region forms $(\sim 25 \mathrm{~nm})$ on the right. The upper panel shows that the resulting band profile exhibits band bending in the inversion and depletion regions and the characteristic sawtooth shape in the active part. The ground state of the quantum well lies far above $(\sim 1 \mathrm{eV})$ the Fermi level (dotted lines). In contrast, the energy level of the 2DEG falls below the Fermi level. In this inversion region, several quantized states exist, but electrons accumulate mostly in the 2DEG of lowest energy. ${ }^{29,34,35}$ The dashed lines indicate the conduction-band profile for an undoped structure for some arbitrary boundary condition. The central role played by the inversion and depletion regions for this simple heterostructure underlines the importance of considering the long-range screening as part of a device design.

\section{B. Tunneling barrier}

The wide depletion barriers significantly reduce tunneling current through a heterostructure. A qualitative WKB (Wentzel-Kramers-Brillouin) analysis serves as an illustration. The WKB tunneling probability is as follows:

$$
T_{\mathrm{tun}}(E) \approx e^{-\int_{\oslash} d z \sqrt{2 m[U(z)-E]} / \hbar}=e^{-L_{\oslash}\langle\sqrt{U-E} / \hbar\rangle_{\oslash}} .
$$

Here $\oslash$ identifies the classically forbidden regions and the angle brackets, an average over the forbidden region. $U$ is the potential energy, $E$ the energy, and $m$ the effective electron mass. The tunneling current is given by the Tsu-Esaki formula, ${ }^{36}$

$$
I\left(V_{\text {bias }}\right)=\frac{2 m}{2 \pi^{2} \hbar^{3}} \int_{0}^{\infty} d E T_{\text {tun }}^{V_{\text {bias }}}(E) D^{V_{\text {bias }}}(E),
$$




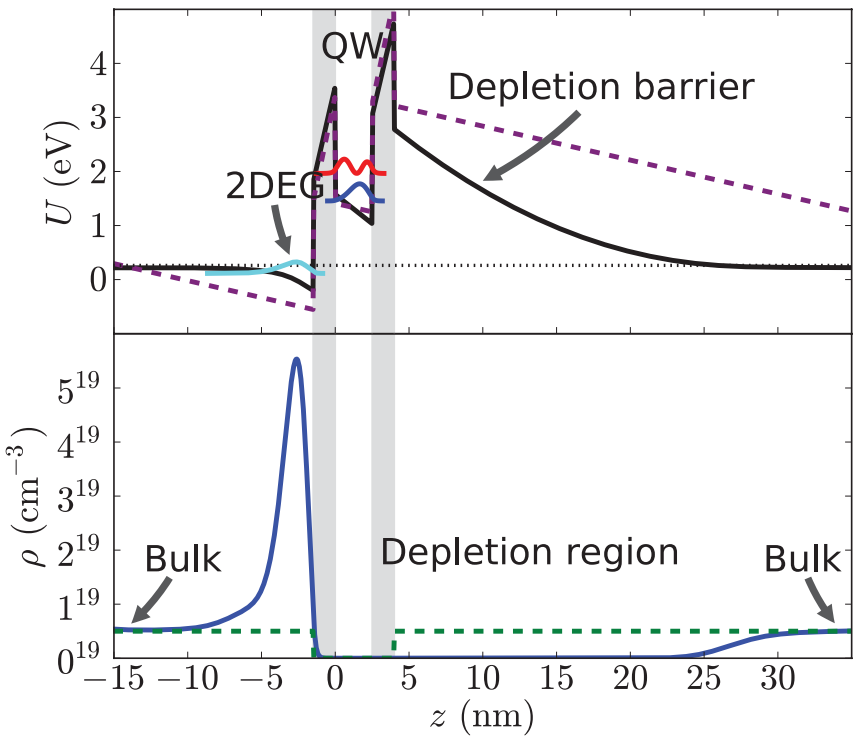

FIG. 1. (Color online) Illustration of transport-inhibiting depletion barriers forming in typical heterostructures composed of AlN and GaN layers. The figure shows conduction-band profile and bound states (upper panel) and charge density (lower panel). The active region consists of 1.5-nm AlN barriers sandwiching a $2.5-\mathrm{nm} \mathrm{GaN}$ layer. Cladding layers consist of doped $\mathrm{GaN}\left(5 \times 10^{18} \mathrm{~cm}^{-3}\right)$. The gray background indicates the AlN layers. In the upper panel the full curves show the conduction-band profile and the dotted indicates the Fermi level. The wavy curves display the computed wave function squared of the three (quasi-)bound states of the structure. Their offsets are given by their energy levels. The dashed lines indicate a possible profile for an undoped heterostructure. In the lower panel, the full curve shows the carrier density, while the dashed lines indicate the background doping density.

$$
D^{V}(E)=\ln \left[\frac{1+\exp \left(\mu_{F}-E\right) / k_{B} T}{1+\exp \left(\mu_{F}-E-V\right) / k_{B} T}\right] .
$$

If $V_{\text {bias }}$ is large, a typical operational condition for AlN/GaN structures, electrons are not Pauli blocked [which enters through the support function $D^{V}(E)$ and Fermi level $\mu_{F}$ ]. The tunneling current is then roughly proportional to the tunneling probability. We make two observations: The heavy mass and large band offset of AlN/GaN reduce the tunneling probability compared to many other materials and the tunneling probability is reduced more by a long classically forbidden region than by a high barrier. In Eq. (2) the term in the exponential increases linearly with the length, while the square root dampens the dependency on the barrier height. Thus, the barrier in the depletion region, which is typically of a wide spatial dimension, but of low energy, has a significant current-impeding effect.

The current-voltage curve for a single AlN barrier with GaN leads is calculated using a transmission coefficient obtained with a transfer-matrix method for arbitrary barrier shapes ${ }^{37}$ and the current $I$ with the Tsu-Esaki equation of Eq. (3). This calculation further corroborates the WKB argument and attests to the extreme current sensitivity to the depletion layer width. The AlN barrier is $1.0 \mathrm{~nm}$ wide (two monolayers). A positive background charge density of $N_{d}=5 \times 10^{18} \mathrm{~cm}^{-3}$ accounts for doping of the GaN leads. The conduction-band

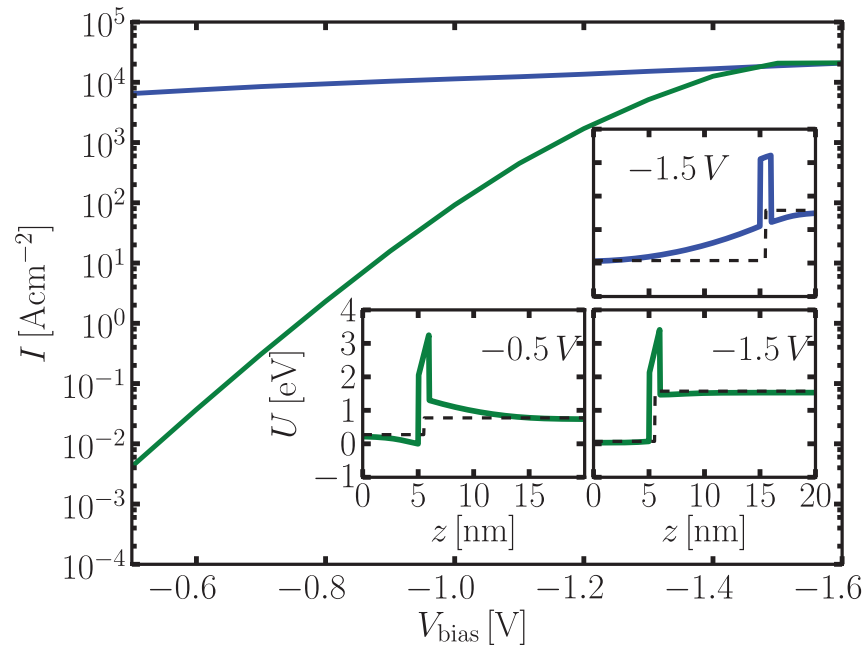

FIG. 2. (Color online) Calculated current-voltage curve for a 1nm-wide AlN barrier with GaN leads. The lower green (upper blue) curve shows the current calculated with spontaneous and piezoelectric polarization included (neglected). The lower insets (upper inset) show the conduction band profile including (neglecting) these effects at a bias of $V_{\text {bias }}=-0.5$ and $-1.5 \mathrm{~V}(-1.5 \mathrm{~V})$. The dashed lines identify the stepped effective Fermi level.

profile $U(x)$ is obtained in an effective-mass SchrödingerPoisson description. ${ }^{16}$ A stepped Fermi level induces the nonequilibrium charge-density distribution.

Figure 2 displays the current-voltage curves for the barrier structure, calculated for conduction-band profiles obtained with and without the polar effects. The current increases enormously with bias until about $-1.4 \mathrm{~V}$, for which the current in the polar case exceeds that of the nonpolar case (which does not exhibit this extreme sensitivity). The extreme bias sensitivity has been also documented in Refs. 15, 28, and 32. The two lower insets show the conduction-band profiles in the polar case for two sample voltages. It shows that for a voltage of $-0.5 \mathrm{~V}$ there is a wide depletion region, while for $-1.5 \mathrm{~V}$, the band is almost flat, with traces of an inversion region influencing the right side. It is evident that the reduction in sensitivity coincides with achieving flat bands in the leads.

The voltage drop arising from the polarization fields is $-1.38 \mathrm{~V}$. Thus, for an applied bias of $V_{\text {bias }}=-1.38 \mathrm{~V}$ the unscreened fields vanish, as the applied bias match the voltage drop over the barrier region. For the single barrier, the conduction band in the lead region, as obtained in the SP calculation, is approximately flat when the applied bias matches the intrinsic voltage drop.

Where the single barrier in a polar heterostructure represents an elementary case of polarization balance, a nonpolar heterostructure is the trivial case; polarization balance is achieved at zero bias. The upper inset in Fig. 2 shows that, in the nonpolar case, the depletion and inversion regions that arise at nonzero bias do not inhibit current as the direction of the slope coincides with the movement of the electrons. ${ }^{38,39}$

\section{Biased double-barrier structures}

Having studied a single barrier, we next study the conduction-band profile of double-barrier structures, arguably 

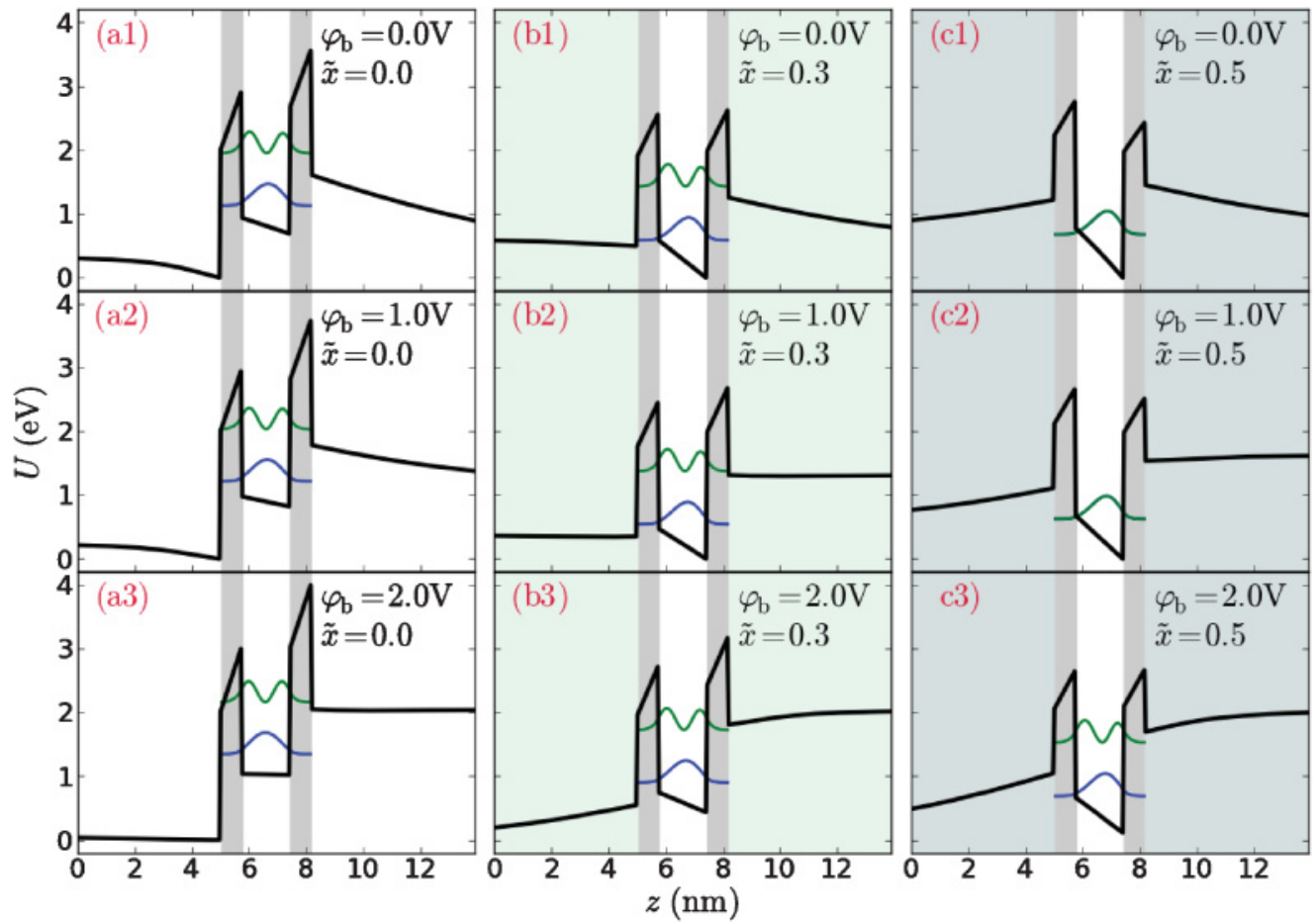

FIG. 3. (Color online) Conduction-band profiles and bound states in quantum well of double-barrier structures with 0.75-nm-wide AlN barriers sandwiching an 1.7-nm-wide GaN layer for varying bias $\varphi_{b}=-V_{\text {bias }}$ and Al concentrations $\tilde{x}$ in the doped $\left(5 \times 10^{18} \mathrm{~cm}^{-3}\right) \mathrm{AlGaN}$ leads.

the simplest structures that exhibit an internal quantum structure within the active region. The intended functionality of the device is electroluminescence. The electrons tunnel resonantly into the upper level of the quantum well, possibly make a transition to the lower level, and exit by tunneling into the continuum. This simplified structure allows us to exemplify design features of complex structures. The few parameters involved in its specification also permit brute-force trend studies, such as that presented in Sec. IV.

In designing many different structures, we found that a typical feature for AlN/GaN structures with GaN leads was that the bias required to overcome the depletion barriers, and, thus, get good transport conditions, was so large that it resulted in poor confinement of the upper level and very hot electrons in the ejection lead. The left panels of Fig. 3 illustrate these issues for a double-barrier structure. It displays the result of a Schrödinger-Poisson calculation for different applied biases, under the assumption of efficient emptying of the active region; the active region and the left lead have the same Fermi level. In panels "a1)" and "a2)," a depletion barrier hinders electron injection. In panel "a3)," the bias is large enough to give flat bands in the leads and within the quantum well. In this case, states in the injection lead resonate with the upper level of the active region. These energy levels match because the AlN layer is appropriately thin for a GaN well of this width. In polar heterostuctures, the energy levels depend strongly on the barrier widths, because of the typically large potential drops across the barrier. The "a3)" structure exhibits troublesome features; the potential of the left barrier is so low that electrons easily escape prior to making a transition, and since the electrons are confined in the triangular part of the barrier, increasing the well width does not help. Moreover, that the electrons leaving the structure are hot can be a problem. For periodic structures, such as QC lasers, electrons may never relax before reaching the next period; it is also inefficient from a power-usage perspective.

Using AlGaN leads with a finite $\mathrm{Al}$ concentration $\tilde{x}$, instead of GaN leads, reduces the potential drop over the active structure at high-transport resonant-tunneling conditions since the unscrened field arises from the difference in polarization between the layers. As the quantum transition occurs mostly within the active structure, the alloy scattering introduced should not severely broaden this peak. The middle and right panels, "bx)" and "cx)," of Fig. 3 show band profiles calculated for alloyed leads. The "b1)" panel shows that for zero bias and $\tilde{x}=0.3$, the depletion region is much reduced compared to $\tilde{x}=0.0$ in "a1)." By increasing the bias, as shown in the "b2" panel, the band in the leads eventually becomes flat, requiring only about half the bias as with GaN leads as shown in panel "a3)." The "b2)" structure confines the upper level much better than if the leads consist of $\mathrm{GaN}$, and the electrons tunneling out of the structure remain fairly cold. Thus, it outperforms that shown in panel "a3)." Both "b2)" and "a3)" are polarization-balanced designs; they operate close to the flat-band condition.

The "c1)" structure highlights another important effect: Here, the energy of the lower level of the active region falls below the conduction band edge of the leads. This results in large band filling, with ensuing depletion regions on both sides of the barrier. Note that for the "c2)" and "c3)" structures, our assumptions about a stepped the Fermi level might be inappropriate. We include these cases for the 
purposes of illustration and completeness. We find that for this particular active structure, the use of $\mathrm{Al}_{0.5} \mathrm{Ga}_{0.5} \mathrm{~N}$ leads [right panels/"cx)"] prohibits flat bands in the leads regardless of applied bias.

The conduction-band profiles of Fig. 3 demonstrate that we can remove the depletion barrier at, and above, a certain bias and that this bias is lowered by using alloyed leads, unless electrons accumulate within the active structure. Since flat bands benefit electron injection, designing structures to operate at this bias is an excellent starting point for making currentdriven devices where high power is essential. A strategy is, therefore, to restrict the design space to those structures that are polarization balanced. The next section develops a theory for conditions that ensures an automatic restriction to such polarization-balanced designs.

\section{POLARIZATION BALANCE}

\section{A. Intrinsic voltage drop}

The voltage drop over a heterostructure arising from polar effects is found by solving Poisson's equation. The solution is

$$
V_{\mathrm{pol}}=\sum_{i=1}^{N}\left(P_{i}-P_{\text {lead }}\right) \frac{L_{i}}{\epsilon_{i}} .
$$

Here $P_{\text {lead }}$ is the polarization of the leads, assumed to be the same in both leads, while $P_{i}$ and $\epsilon_{i}$ are the polarization and dielectric constant of layer $i$. The polarization of a layer has both spontaneous and piezoelectric components: $P_{i}=P_{i}^{\mathrm{SP}}+$ $P_{i}^{\text {piezeo }}$.

For systems with a slowly varying composition or lattice constant, an integral formulation is more appropriate, as follows:

$$
V_{\text {pol }}=\int_{\text {left lead }}^{\text {right lead }} d z\left[P(z)-P_{\text {lead }}\right] \frac{1}{\epsilon(z)} .
$$

Naturally, this expression reduces to Eq. (5) for discrete variations of composition and lattice constant. Equations (5) and (6) determine the intrinsic voltage drop under broad conditions. Polarization balance is obtained when it equals the applied bias, $V_{\text {pol }}=V_{\text {bias }}^{0}$. This condition generally requires that the two lead compositions are the same; otherwise, interface charges do not cancel out, and the unscreened fields cannot vanish in the lead region.

If the active region is fully or partially relaxed, the lead regions on both sides should be defined so they have the same lattice constant and, thereby, piezoelectric contributions.

\section{B. Heterostructures of two-component alloys: AIN/AIGaN/GaN}

The expression for intrinsic voltage drop is further developed here for heterostructures with layers having alloy concentrations within the same class of binary alloys. We use AlN/AlGaN/GaN layers as a specific case, but the expressions can be applied to other material systems. Here, Vegard's law determines the spontaneous polarization, $P^{\mathrm{sp}}(w)=$ $P_{\mathrm{GaN}}^{\mathrm{sp}}(1-w)+P_{\mathrm{AlN}}^{\mathrm{sp}} w$, the bulk lattice constant, $a_{\mathrm{bulk}}(w)=$ $(1-w) a_{\mathrm{GaN}}+w a_{\mathrm{AlN}}$, as well as other basic parameters of $\mathrm{Al}_{w} \mathrm{Ga}_{1-w} \mathrm{~N}$. The polarization-balance concept is not restricted to this approximation, but computations are simpler and it allows for handy equations to be developed. Vegard's law is, for these quantities, a fair approximation for AlGaN layers, but it is less appropriate for alloys with larger bowing parameters, such as InGaN and InAlN. ${ }^{40}$

The piezoelectric polarization can be expressed as

$$
P_{i}^{\text {piezo }}\left(y_{i}, x_{i}\right)=y_{i} \Gamma^{A}\left(x_{i}\right)-\Gamma^{B}\left(x_{i}\right),
$$

where $x_{i}$ is the alloy concentration of the layer and $y_{i}$ is identified by the bulk alloy concentration corresponding to the lattice constant of the layer, that is,

$$
a_{\text {bulk }}\left(y_{i}\right)=a_{i} \text {. }
$$

The heterostructure may have a common lattice constant, $x_{i}=$ $x^{\prime}$. The piezoelectric material parameters $\Gamma^{A}$ and $\Gamma^{B}$ are

$$
\begin{aligned}
& \Gamma^{A}(w)=\left[a_{\mathrm{AlN}}-a_{\mathrm{GaN}}\right] \frac{2 D(w)}{a(w)}, \\
& \Gamma^{B}(w)=\left[a(w)-a_{\mathrm{GaN}}\right] \frac{2 D(w)}{a(w)} .
\end{aligned}
$$

Here $D(w)=e_{31}(w)-e_{33}(w) c_{13}(w) / c_{33}(w)$, with $c_{13}$ and $c_{33}$ being elastic coefficients and $e_{31}$ and $e_{33}$ piezoelectric coefficients. ${ }^{27}$ In particular, we identify $\Gamma^{A}(0) \equiv \Gamma_{\mathrm{GaN}}$, $\Gamma^{B}(0) \equiv 0$, and $\Gamma^{A}(1)=\Gamma^{B}(1) \equiv \Gamma_{\text {AlN }}$. Figure 4 displays $\Gamma_{A}$ and $\Gamma_{B}$ as a function of alloy concentration $w$. While $\Gamma_{A}$ is almost linear, $\Gamma_{B}$ exhibits clear bowing. A linear approximation for this term is inaccurate.

With the notation introduced here, we obtain

$$
\begin{gathered}
V_{\mathrm{pol}}=\sum_{i=1}^{N-1}\left[P_{i}^{\mathrm{sp}}+x_{i} \Gamma_{i}^{A}-\Gamma_{i}^{B}-P_{\text {lead }}\right] \frac{L_{i}}{\epsilon_{i}}, \\
P_{\text {lead }}=-\tilde{x} P_{\mathrm{AlN}}^{\mathrm{sp}}-(1-\tilde{x}) P_{\mathrm{GaN}}^{\mathrm{sp}}+\tilde{y} \Gamma^{A}(\tilde{x})-\Gamma^{B}(\tilde{x}) .
\end{gathered}
$$

Here $\tilde{x}$ is the alloy concentration of the leads and $\tilde{y}$ is the bulk $\mathrm{AlGaN}$ concentration corresponding to the lattice constant of the leads [Eq. (8)].

Two consistency checks of Eq. (10) are readily available: First, if a layer (for instance, the leads) is relaxed, $x_{i}=$ $y_{i}$, its piezoelectric component, $P_{\text {piezo }}\left(x_{i}, x_{i}\right)=x_{i} \Gamma^{A}\left(x_{i}\right)-$ $\Gamma^{B}\left(x_{i}\right)=0$, vanishes. Second, the intrinsic voltage drop does

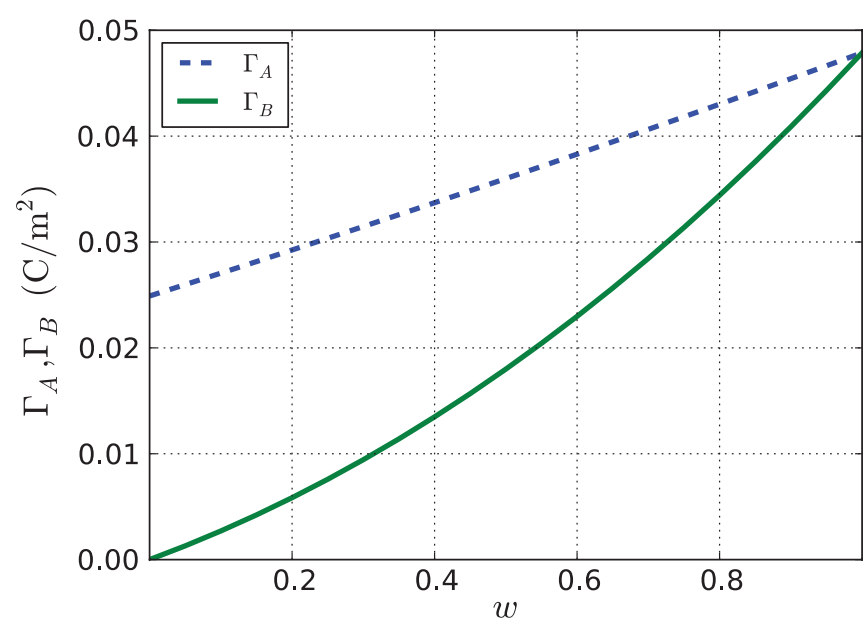

FIG. 4. (Color online) Piezoelectric material parameters $\Gamma_{A}$ and $\Gamma_{B}$ as a function of the alloy concentration of an $\mathrm{Al}_{w} \mathrm{Ga}_{1-w} \mathrm{~N}$ layer as given by Eq. (9). 
TABLE I. Polarization-related material quantities introduced here based on the parameters of Ref. 41 .

\begin{tabular}{cccc}
\hline \hline$A(\mathrm{~V} / \AA)$ & $B(\mathrm{~V} / \AA)$ & $\Gamma_{\mathrm{AIN}}\left(\mathrm{C} / \mathrm{m}^{2}\right)$ & $\Gamma_{\mathrm{GaN}}\left(\mathrm{C} / \mathrm{m}^{2}\right)$ \\
\hline-0.138 & -0.094 & 0.048 & 0.025 \\
\hline \hline
\end{tabular}

not depend on the starting point of the lead region: If we define part of a lead (layer $k$ ) as a layer within the active region, the intrinsic potential drop $V_{\text {pol }}$ remains the same, because the additional $k$ term yields zero: $P_{k}-P_{\text {lead }}=0$.

\section{AIN/GaN layers strained to AIGaN leads}

If the active region is composed of $\mathrm{AlN}$ and GaN layers, and these are strained to the leads $\left(x^{\prime}=\tilde{x}=\tilde{y}\right)$, there is a simple relation between the lead alloy concentration $\tilde{x}$, intrinsic voltage drop over active region length $V_{\text {pol }} / L$, and average concentration in the active region as follows:

$$
x=\sum L_{i}^{\mathrm{AlN}} / L \text {. }
$$

The relation can be stated as follows:

$$
\tilde{x}=\frac{-V_{\mathrm{pol}} / L+A x}{A x+B(1-x)} .
$$

The material specific values $A$ and $B$ are given by

$$
\begin{aligned}
& A=\left(P_{\mathrm{sp}}^{\mathrm{AlN}}-P_{\mathrm{sp}}^{\mathrm{GaN}}-\Gamma^{\mathrm{AlN}}\right) \frac{1}{\epsilon_{\mathrm{AlN}}}, \\
& B=\left(P_{\mathrm{sp}}^{\mathrm{AlN}}-P_{\mathrm{sp}}^{\mathrm{GaN}}-\Gamma^{\mathrm{GaN}}\right) \frac{1}{\epsilon_{\mathrm{GaN}}},
\end{aligned}
$$

with materials-specific values listed in Table I. In deriving the relation (12) between intrinsic voltage drop and lead concentration, we have used the Vergard's law as in the previous subsection.

The counter plot of Fig. 5 shows $V_{\text {pol }} / L$ as function of the average composition $x$ and lead concentration $\tilde{x}$. By setting the applied bias according to these contours, we get polarization balance, that is, we remove the unscreened fields in the leads.

We confirm the consistency of Eq. (12) by showing that two polarization-balanced active regions are also naturally described as a single polarization-balanced region. We consider two active regions with average compositions $x_{1}$ and $x_{2}$, of lengths $L_{1}$ and $L_{2}$, producing respectively an intrinsic voltage drop of $V_{1}, V_{2}$ for the same lead alloy concentration $\tilde{x}$. We test whether $\tilde{x}$ is the solution to the following equation (with unknown $z$ ) describing the two regions in terms of a single active region,

$$
\begin{aligned}
& -\frac{V_{1}+V_{2}}{L_{1}+L_{2}}+A \frac{x_{1} L_{1}+x_{2} L_{2}}{L_{1}+L_{2}} \\
& \quad=z\left[A \frac{x_{1} L_{1}+x_{2} L_{2}}{L_{1}+L_{2}}+B\left(1-\frac{x_{1} L_{1}+x_{2} L_{2}}{L_{1}+L 2}\right)\right] .
\end{aligned}
$$

Singling out the $L_{1}$ and $L_{2}$ terms, we obtain

$$
\begin{aligned}
& L_{1}\left[-V_{1} / L_{1}+A x_{1}-z\left(A x_{1}+B\left(1-x_{1}\right)\right)\right] \\
& \quad+L_{2}\left[-V_{2} / L_{2}+A x_{2}-z\left(A x_{2}+B\left(1-x_{2}\right)\right)\right]=0 .
\end{aligned}
$$

Since $z=\tilde{x}$ is a solution, the two descriptions are equivalent.

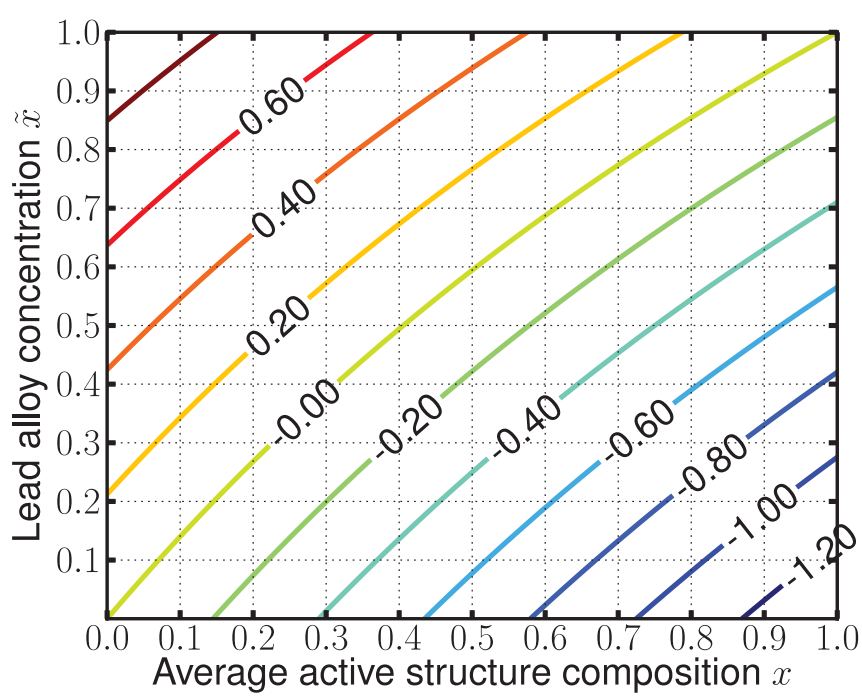

FIG. 5. (Color online) The intrinsic voltage drop per length $V_{\text {pol }} / L(\mathrm{~V} / \mathrm{nm})$ of the active region as a function of the average composition $x$ of the active region and the alloy concentration of leads $\tilde{x}$. The active region is strained to the leads.

\section{EXAMPLES OF APPLICATIONS}

\section{A. Double-barrier structure polarization balanced for zero bias}

Figure 6 displays the conduction-band profile and bound states of an unbiased structure composed of a GaN quantum well between the AlN barriers and AlGaN cladding layers with alloy concentraction chosen for polarization balance at zero bias. There is a significant band filling of the active region that generates depletion regions on both sides of the active region. This contrasts the depletion and inversion regions outside the

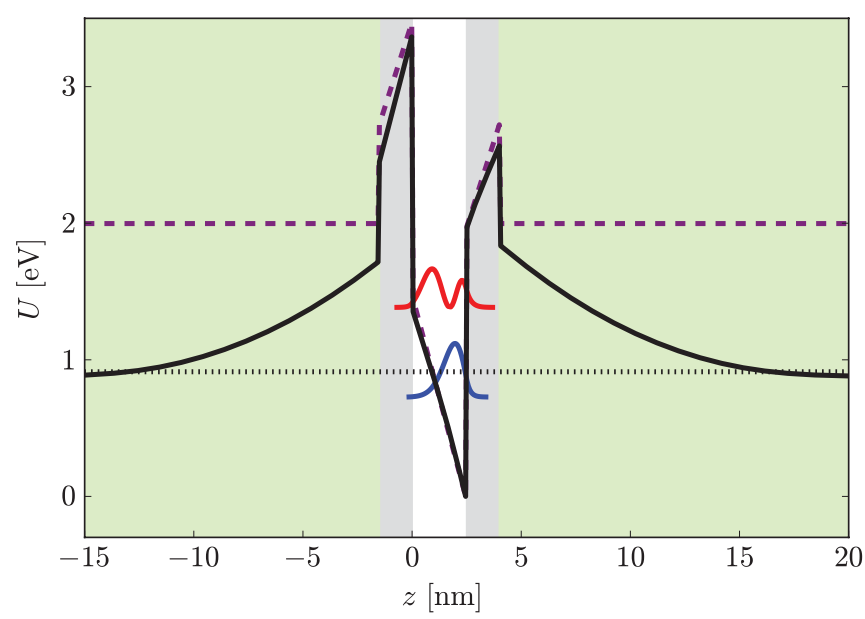

FIG. 6. (Color online) Conduction-band profile and bound states of an optically active quantum well with a polarization-balanced design for zero bias. It consists of 2.5-nm-wide AlN barriers, a 1.5-nm-wide GaN well layer, and cladding layers consisting of $\mathrm{Al}_{0.63} \mathrm{Ga}_{0.37} \mathrm{~N}$. Cladding layers are doped to $5 \times 10^{18} \mathrm{~cm}^{-3}$. The light gray (green) background indicates $\mathrm{AlGaN}$, whereas the white and gray background indicates $\mathrm{GaN}$ and $\mathrm{AlN}$, respectively. The dashed (purple) lines show the band profile of an undoped structure. The wavy curves indicate the wave function squared of the bound states offseted by their energy. The dotted line represents the Fermi level. 
depleted active region for GaN cladding layers, as displayed in Fig. 1 and the "1a)" panel of Fig. 3. The dashed lines confirm the polarization-balance equations, giving a flat band in the cladding layers for an undoped structure.

The elimination of unscreened fields in the cladding layers (polarization balance) causes charging of the active region for unbiased structures because the energy of the lowest quantum well state falls below the condition band edge. The band bending in the cladding layers underlines that merely fulfilling polarization balance does not necessarily result in a flat band. In the unbiased case polarization balance instead ensures efficient filling of the active region rather than of the 2DEG at the interface. This result relates to an earlier observation that, by using the same alloy composition in the active region as in the cladding layers, good control over the electron population can be achieved. ${ }^{2,17}$

\section{B. Design of quantum-cascade structure}

If an active region is approximately charge neutral at operating conditions, the polarization-balanced design succeeds in removing depletion and inversion regions. This could be the case for heterostructures with efficient emptying of the active region. By adjusting the bias over a structure, we can always make a structure polarization balanced; however, the required bias can be unsuited for meaningful designs of the active structure. This was illustrated for GaN leads in our trial-and-error study detailed in Fig. 3.

The additional freedom of design gained by using alloyed leads for polarization-balanced structures is demonstrated with a large parameter study of a simple two-level quantum- cascade (QC) structure. The active region consists of two AIN barriers sandwiching a $\mathrm{GaN}$ well as in previously studied structures. We vary well $\left(L_{w}\right)$ and barrier $\left(L_{\text {bar }}\right)$ widths and lead alloy concentration $\tilde{x}$. The bias is always adjusted to obtain polarization balance. In calculating energies and wave functions, we consider only the active region. This is because we found only a tiny difference between the band profile as obtained with and without doping of the leads, given that charging of the active region is avoided.

The merits of a design depend on the intended application; for instance, gain and threshold current are key quantities for lasers. Rather than considering an application-specific assessment of our QC structure, we consider three intuitive, but somewhat crude, design criteria. The first is injection of the upper level: The energy should lie above the conduction band edge of the injection lead but no more than $200 \mathrm{meV}$ above. This upper bound is somewhat arbitrary; in practice it depends on the band filling of the leads and level broadening. However, the applied bias can be adjusted to accommodate slight misalignment. The second is confinement of the upper level: The energy should fall below the triangular portion of the barrier to increase the fraction of the electrons making a transition to the lower level by avoiding the faster FowlerNordheim tunneling. The third is ejection of the lower level: The energy should lie above the conduction band edge of the exit lead. Efficient ejection is essential to avoid charge buildup causing depletion regions in the injection lead and Pauli blocking of optical transitions. Finally, we aim to design a structure operating at the fiberoptic frequency of $\sim 800 \mathrm{meV}$.

Figure 7 details the results of our large-parameter study of the simple QC structure. It shows that the structures with GaN

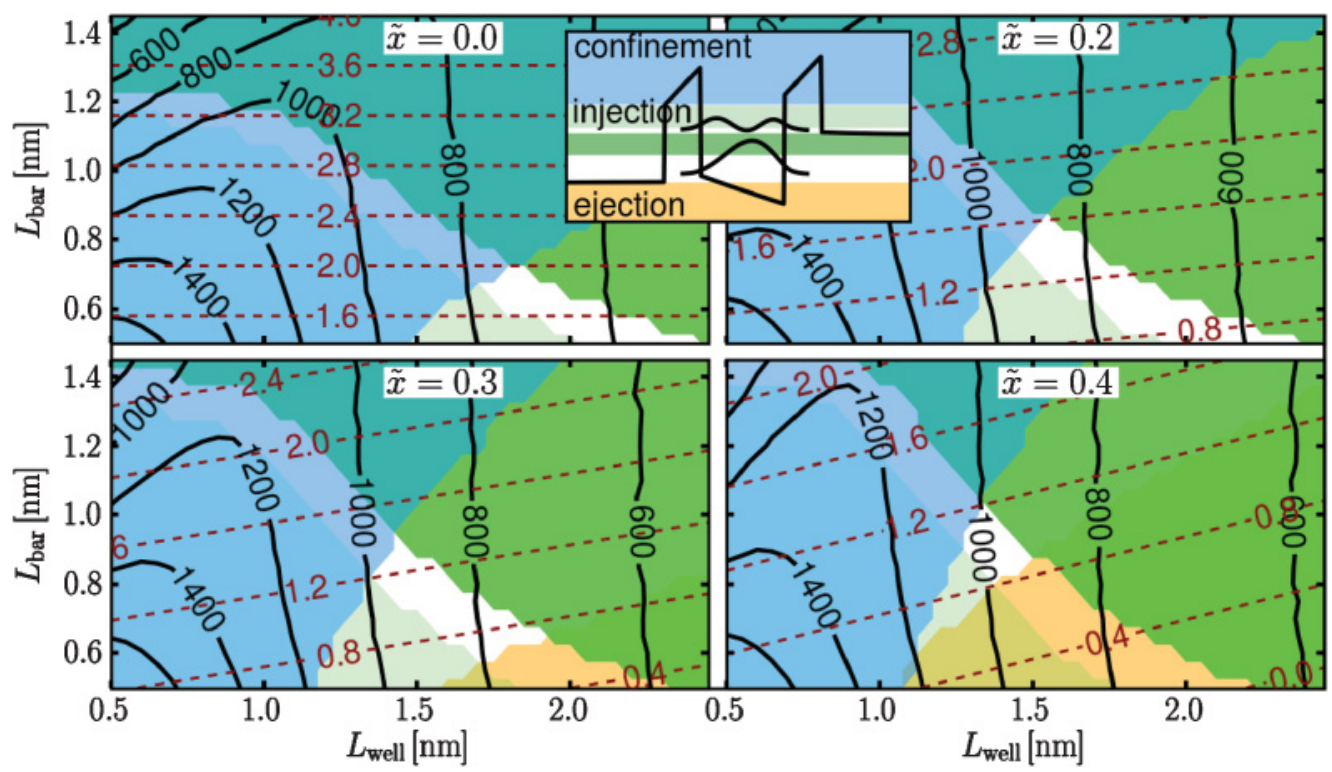

FIG. 7. (Color) Design criteria, transition energy, and applied bias contours for a polarization-balanced two-level QC structure as a function of well and barrier widths for different $\mathrm{Al}_{\tilde{x}} \mathrm{Ga}_{1-\tilde{x}} \mathrm{~N}$ alloy concentrations of the leads, $\tilde{x}=0,0.2,0.3$, and 0.4 . The numbers along the dashed curves denote the absolute value of applied bias [ $V_{\text {bias }}(V)$ ], set to achieve polarization balance [using Eq. (12)], whereas those on the solid line denote the transition energy $\Delta E_{12}(\mathrm{meV})$ between the two levels in the well. The white areas indicate where all design criteria are met; the shaded semitransparent areas indicate where they are not; the blue areas indicate where confinement of the upper level is poor. The yellow areas indicate where ejection of the lower level is problematic. In the green (light green) areas, the injection is problematic because the energy of the upper level falls below (far above) the band edge of the injection lead. The inset shows the band profile and the absolute square of the localized wave functions offset by their energy for the simple QC structure, and the shaded regions indicates design criteria. 
leads does not fulfill the design criteria for a transition energy of $800 \mathrm{meV}$. The bias over the structure is also generally about twice the transition energy, causing ultrahot electrons to exist in and around the structure. For larger alloy concentrations in the leads, the acceptable parameter window moves to wider barriers and thinner wells. For $\tilde{x}=0.2$ and $\tilde{x}=0.3$, all design targets can be accommodated for the fiberoptic frequency. When increasing lead alloy concentrations further, the allowed window contracts, and, for $\tilde{x}=0.5$, no parameters support all design criteria.

\section{DISCUSSION}

\section{A. Conduction-band regimes}

Figure 8 provides an overview of the different structures and bias conditions considered in this study and attempts to sort these into different conduction-band regimes. The horizontal-axis indicates different applied bias over the structure. The vertical-axis represents an abstract structure space; for instance, increasing the $\mathrm{Al}$ concentration of the cladding layer for a given active structure corresponds to moving downward. The (blue) curve denotes a structure in polarization balance. The gray (orange) region indicates that electrons accumulate within the active region, with ensuing depletion barriers on both sides of the active region.

We identify the position of structures studied. In Fig. 1, we considered a structure far from polarization balance and with an empty active region. It exhibited a wide depletion region on the right side of the structure and a well-filled inversion region on the left. The panels of Fig. 3 displayed three different structures under three different voltage conditions. The structures under varying bias are indicated by the three semitransparent axes. Two of the panels ["a3)" and "b2)"] showed structures under a bias that made them approximately polarization

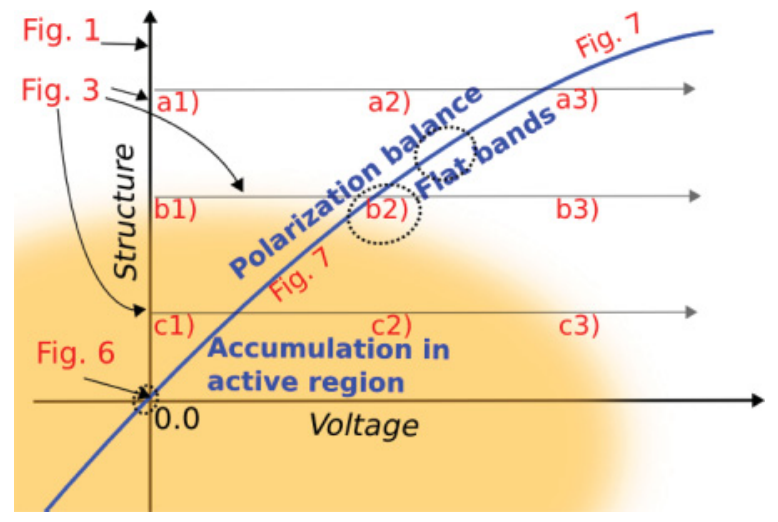

FIG. 8. (Color online) Overview of considered structures: Different conduction-band regimes as a function of applied voltage (horizontal axis) and an abstract structure space (vertical axis). The red curve denotes polarization-balance conditions. For structures along this curve, the lead bands are flat unless charge accumulates within the active region, which happens where the curve enters the gray (orange) region. The figure numbers of structures considered in this study are denoted on various spots. All cases considered in Fig. 7 are polarization balanced. Those within the dashed circles fulfill certain chosen design conditions. This makes them polarizationbalanced designs. balanced. In two of the structures, much charge accumulates within the active region ["c1)" and "c2)"] as the Fermi level was above the lowest level of the active region. The structure showed in Fig. 6 was designed for polarization balance at zero bias according to the theory developed in Sec. III. This secures excellent charge accumulation in the active region. Figure 7 displayed key results of numerous structures at a bias that gives polarization balance. In Fig. 8 all these results scatter along the curve, indicating polarization balance. Some of the designs resulted in filling of the active region [placing them within the gray (orange) region] and are, therefore, among the failed designs. The purpose of the dashed circles is to indicate (in an abstract sense) successful polarization-balanced designs according to the selected design criteria.

The placement of the results of the single barrier displayed in Fig. 2 are not shown here. We note that since the single barrier does not have any internal structure, accumulation within the active structure does not occur. A single barrier cannot be designed for polarization balance at zero bias. The nonpolar case is always polarization balanced at zero bias, but for such structures, polarization balance does not guarantee charge accumulation within the active region.

\section{B. Prospects and challenges}

The theory developed for polarization balance may be useful for designing structures not intended as polarizationbalanced designs, since it can also be used to identify the flat-band condition. Designing a device to operate at an applied bias larger than that resulting in polarization balance may be advantageous for some purposes, for instance, to ensure accumulation layers in the injection lead, making its operation more like that of a nonpolar structure. ${ }^{42}$ Nevertheless, if an effective continuum should arise in the injection region, the bias across it should be small to avoid the formation of a Stark ladder. ${ }^{43,44}$

The huge strain in the structures considered in this study might render them unsuitable for actual applications, although there have been numerous attempts to make resonant-tunneling diodes using GaN leads that have even larger strains. ${ }^{18-20,22,23}$ Using AlGaInN layers could be helpful but complicates growth. With this additional criterion, one could design structures that are both polarization and strain balanced. ${ }^{24}$ Only in special accidental cases can one fulfill both these criteria using layers within the same class of binary alloys (such as AlGaN).

Lattice matching by using AlInN barriers and GaInN wells $^{45}$ removes strain completely. However, with only two different alloy compositions of the layers the generic polarization balance approach is impossible, since it in general requires layers of at least three different compositions. We therefore mention that polarization-balance concepts can also be developed for leads that are superlattices. Such an approach is in the spirit of digital alloys. It would allow for structures that are both polarization balanced and lattice matched (or the more relaxed strain-balance condition), for instance, by combining $\mathrm{Al}_{82} \mathrm{In}_{18} \mathrm{~N}$ and GaN. ${ }^{46,47}$ Since strain is zero, we balance only the spontaneous part of the polarization.

Aside from presenting a solution to the current inhibiting effect of band tailing in the depletion region, polarizationbalanced designs present an additional advantage: The shape of the conduction band does not depend on inversion and 
depletion regions. This has the consequence that the conduction-band profile essentially decouples from the specific doping and carrier concentration. This reduces the importance of an exact control over the donor density for the functionality of a device, which is also helpful since the number of ionized donors is complicated to calculate in heterostructures or when the binding energy is similar to the thermal energy. ${ }^{48}$ We note that in our quasistatic study, effects like charge trapping and formation of field domains ${ }^{43}$ are not included, and these could complicate design considerations.

For emission structures of short optical wavelength, and in particular for QC lasers composed of an alternating series of optically active and interjecting current injection regions, the possibility of directly engineering zero-bias conditions in the injection region (or in the leads) points to an additional strong advantage of polar heterostructures with polarization-balanced designs. In contrast to conventional nonpolar structures, it is not necessary to grade the injection layers to compensate for the field. A large applied bias does not inevitably result in a large electrical fields in the leads or, for that matter, in the entire active (transport-limiting) region. In a good design, the potential drop can be engineered to occur primarily in the region where the optical transition takes place. We hypothesize that this advantage can result in a significant simplification of the tailoring of the quantum wells and barriers that ensures efficient injection in devices like QC lasers.

\section{CONCLUSION}

The polarization-balance concept represents a guiding principle for robust designs of wurtzite AlN/GaN heterostructures grown in the $c$ direction. For high-power current-driven devices, it helps to eliminate the depletion barrier that impedes the perpendicular current. It also simplifies design since one can, to a larger extent, focus on the active structure. This is because the influence of the doping levels on the conductionband profile is substantially reduced as the conduction band is flat in the leads. Furthermore, the flat bands in the leads makes grading of injection regions in QC lasers unnecessary. For such designs of unbiased structures it instead secures a large electron population in the active region.

Our study has been restricted to AlGaN, since the large mass and band offset accentuates the current inhibiting effect of the depletion regions. However, the concept naturally generalizes not only to nitrides, ${ }^{47}$ including $\mathrm{InN}$ and $\mathrm{BN}$ and their alloys, but also to heterostructures of other polar materials such as $\mathrm{ZnMgO} / \mathrm{ZnO}{ }^{49}$

\section{ACKNOWLEDGMENT}

We thank F. Capasso and T. Ive for useful discussions. SNIC is acknowledged for supporting K.B.'s participation in the National Graduate School in Scientific Computing (NGSSC). Vinnova (Banbrytande IKT) provided financial support.
${ }^{1}$ M. C. Amann, F. Capasso, A. Larsson, and M. Pessa, New. J. Phys. 11, 125012 (2009).

${ }^{2}$ H. Machhadani, P. Kandaswamy, S. Sakr, A. Vardi, A. Wirtmüller, L. Nevou, F. Guillot, G. Pozzovivo, M. Tchernycheva, A. Lupu et al., New. J. Phys. 11, 125023 (2009).

${ }^{3}$ J.-S. Yang, H. Sodabanlu, M. Sugiyama, Y. Nakano, and Y. Shimogaki, J. Cryst. Growth 314, 252 (2011).

${ }^{4}$ M. Tchernycheva, L. Nevou, L. Doyennette, F. H. Julien, E. Warde,

F. Guillot, E. Monroy, E. Bellet-Amalric, T. Remmele, and M. Albrecht, Phys. Rev. B 73, 125347 (2006).

${ }^{5}$ V. D. Jovanović, Z. Ikonić, D. Indjin, P. Harrison, and R. A. Soref, Jpn. J. Appl. Phys. 43, 7444 (2004).

${ }^{6}$ K. Kishino, A. Kikuchi, H. Kanazawa, and T. Tachibana, Appl. Phys. Lett. pp. 1234 (2002).

${ }^{7}$ L. B. Cen, B. Shen, Z. X. Qin, and G. Y. Zhang, J. Appl. Phys. 105, 053106 (2009).

${ }^{8}$ A. Ishida, K. Matsue, Y. Inoue, H. Fujiyasu, H.-J. Ko, A. Setiawan, J.-J. Kim, H. Makino, and T. Yao, Jpn. J. Appl. Phys. 44, 5918 (2005).

${ }^{9}$ N. Vukmirović, V. D. Jovanović, D. Indjin, Z. Ikonić, P. Harrison, and V. Milanović, J. Appl. Phys. 97, 103106 (2005).

${ }^{10}$ W. Terashima and H. Hirayama, Phys. Status Solidi C 6, S615 (2009).

${ }^{11}$ M. Stattin, K. Berland, P. Hyldgaard, A. Larsson, and T. G. Andersson, Phys. Status Solidi C 8, 2357 (2011).

${ }^{12}$ F. Bernardini and V. Fiorentini, Phys. Rev. B 57, R9427 (1998).

${ }^{13}$ V. Fiorentini, F. Bernardini, F. Della Sala, A. Di Carlo, and P. Lugli, Phys. Rev. B 60, 8849 (1999).

${ }^{14}$ F. Sudradjat, W. Zhang, K. Driscoll, Y. Liao, A. Bhattacharyya, C. Thomidis, L. Zhou, D. J. Smith, T. D. Moustakas, and R. Paiella, J. Appl. Phys. 108, 103704 (2010).
${ }^{15}$ M. Hermann, E. Monroy, A. Helman, B. Baur, M. Albrecht, B. Daudin, O. Ambacher, M. Stutzmann, and M. Eickhoff, Phys. Status Solidi C 1, 2210 (2004).

${ }^{16}$ K. Berland, M. Stattin, R. Farivar, D. M. S. Sultan, P. Hyldgaard, A. Larsson, S. M. Wang, and T. G. Andersson, Appl. Phys. Lett. 97, 043507 (2010).

${ }^{17}$ M. Tchernycheva, L. Nevou, L. Vivien, F. H. Julien, P. K. Kandaswamy, E. Monroy, A. Vardi, and G. Bahir, Phys. Status Solidi B 247, 1622 (2010).

${ }^{18}$ A. Kikuchi, R. Bannai, K. Kishino, C.-M. Lee, and J.-I. Chyi, Appl. Phys. Lett. 81, 1729 (2002).

${ }^{19}$ A. E. Belyaev, O. Makarovsky, D. J. Walker, L. Eaves, C. T. Foxon, S. V. Novikov, L. X. Zhao, R. I. Dykeman, S. V. Danylyuk, S. A. Vitusevich et al., Physica. E 21, 752 (2004).

${ }^{20}$ S. Golka, C. Pflügl, W. Schrenk, G. Strasser, C. Skierbiszewski, M. Siekacz, I. Grzegory, and S. Porowski, Appl. Phys. Lett. 88, 172106 (2006).

${ }^{21}$ C. Bayram, Z. Vashaei, and M. Razeghi, Appl. Phys. Lett. 97, 181109 (2010).

${ }^{22}$ C. Bayram, Z. Vashaei, and M. Razeghi, Appl. Phys. Lett. 96, 042103 (2010).

${ }^{23}$ Z. Vashaei, C. Bayram, and M. Razeghi, J. Appl. Phys. 107, 083505 (2010).

${ }^{24}$ V. D. Jovanović, Z. Ikonić, D. Indjin, P. Harrison, V. Milanović, and R. A. Soref, J. Appl. Phys. 93, 3194 (2003).

${ }^{25} \mathrm{At}$ times we will use the symbol $\varphi_{b}=-V_{\text {bias }}$ rather than the applied bias $V_{\text {bias }}$ for convenience; using the standard convention for the $z$-axis, we always consider an applied bias that is nonpositive and a particle current going from right to left.

${ }^{26}$ J. Faist, F. Capasso, D. L. Sivco, A. L. Hutchinson, C. Sirtori, S. N. G. Chu, and A. Y. Cho, Appl. Phys. Lett. 65, 2901 (1994). 
${ }^{27}$ S. Gunna, F. Bertazzi, R. Paiella, and E. Bellotti, in Nitride Semiconductor Devices: Principles and Simulation (Weinheim-VCH Verlag Gmbh \& Co. KGaA, Weinheim, 2007).

${ }^{28}$ S. Leconte, F. Guillot, E. Sarigiannidou, and E. Monroy, Semicond. Sci. Technol. 22, 107 (2007).

${ }^{29}$ K. Berland, Superlattices Microstruct. 50, 411 (2011).

${ }^{30}$ C. Gmachl, H. M. Ng, and A. Y. Cho, Appl. Phys. Lett. 79, 1590 (2001).

${ }^{31}$ X. Y. Liu, P. Holmström, P. Jänes, L. Thylén, and T. G. Andersson, Phys. Status Solidi B 244, 2892 (2007).

${ }^{32}$ S. Sakr, E. Warde, M. Tchernycheva, and F. H. Julien, J. Appl. Phys. 109, 023717 (2011).

${ }^{33}$ O. Ambacher, J. Smart, J. R. Shealy, N. G. Weimann, K. Chu, M. Murphy, W. J. Schaff, L. F. Eastman, R. Dimitrov, L. Wittmer et al., J. Appl. Phys. 85, 3222 (1999).

${ }^{34}$ T. Ando, A. B. Fowler, and F. Stern, Rev. Mod. Phys. 54, 437 (1982).

${ }^{35}$ A. A. Klochikhin, V. Y. Davydov, I. Y. Strashkova, and S. Gwo, Phys. Rev. B 76, 235325 (2007).

${ }^{36}$ R. Tsu and L. Esaki, Appl. Phys. Lett. 22, 562 (1973).

${ }^{37}$ Y. Ando and T. Itoh, J. Appl. Phys. 61, 1497 (1987).

${ }^{38}$ M. Cahay, M. McLennan, S. Datta, and M. S. Lundstrom, Appl. Phys. Lett. 50, 612 (1987).
${ }^{39}$ E. R. Brown, Hot Carriers in Semiconductor Nanostructures (Academic Press, New York, 1992), p. 469.

${ }^{40}$ F. Bernardini and V. Fiorentini, Phys. Rev. B 64, 085207 (2001).

${ }^{41}$ I. Vurgaftman and J. R. Meyer, Nitride Semiconductor Devices: Principles and Simulation (2007), pp. 13-48.

${ }^{42}$ M. Rahman and J. H. Davies, Semicond. Sci. Technol. 5, 168 (1990).

${ }^{43}$ A. Wacker, Phys. Rep. 357, 1 (2002).

${ }^{44}$ L. Esaki and L. L. Chang, Phys. Rev. Lett. 33, 495 (1974).

${ }^{45}$ T. Lim, R. Aidam, L. Kirste, P. Waltereit, R. Quay, S. Müller, and O. Ambacher, Appl. Phys. Lett. 96, 252108 (2010).

${ }^{46}$ M. Gonschorek, J.-F. Carlin, E. Feltin, M. A. Py, and N. Grandjean, Appl. Phys. Lett. 89, 062106 (2006).

${ }^{47}$ D. Jena, J. Simon, A. K. Wang, Y. Cao, K. Goodman, J. Verma, S. Ganguly, G. Li, K. Karda, V. Protasenko et al., Phys. Status Solidi A 208, 1511 (2011).

${ }^{48}$ P. Van Mieghem, Rev. Mod. Phys. 64, 755 (1992).

${ }^{49}$ H. Tampo, H. Shibata, K. Maejima, A. Yamada, K. Matsubara, P. Fons, S. Kashiwaya, S. Niki, Y. Chiba, T. Wakamatsu et al., Appl. Phys. Lett. 93, 202104 (2008). 